\title{
Impact of the COVID-19 epidemic at a high-volume facility in gynecological oncology in Tokyo, Japan: a single-center experience
}

Yuya Nogami, Yusuke Kobayashi* ${ }^{*}$, Kosuke Tsuji, Megumi Yokota, Hiroshi Nishio, Masaru Nakamura, Wataru Yamagami, Tohru Morisada, Eiichiro Tominaga, Kouji Banno and Daisuke Aoki

\begin{abstract}
Background: The number of cases of novel coronavirus disease 2019 (COVID-19) in Japan have risen since the first case was reported on January 24, 2020, and 6225 infections have been reported as of June 30, 2020. On April 8, 2020, our hospital began screening patients via pre-admission reverse transcriptase-polymerase chain reaction (RTPCR) for severe acute respiratory syndrome coronavirus 2 (SARS-CoV-2) and chest computed tomography (CT). Although no patients exhibited apparent pneumonia, treatment delay or changes in treatment plans were required for a few patients based on the results of screening tests. During an emerging infectious disease pandemic, the likelihood of being infected, as well as the disease itself, affects clinical decision making in several ways. We summarized and presented our experience.

Case presentation: After the introduction of pre-admission screening, RT-PCR and CT were performed in 200 and 76 patients, respectively, as of June 30,2020. The treatment of five patients, including two patients with cervical cancer, two patients with ovarian tumors, and one patient with ovarian cancer, was affected by the results. Two asymptomatic RT-PCR-positive patients did not develop COVID-19, but their treatment was delayed until the confirmation of negative results. The other three patients were RT-PCR-negative, but abnormal CT findings suggested the possibility of COVID-19, which delayed treatment. The patients receiving first-line preoperative chemotherapy for ovarian cancer had clinically evident exacerbations because of the treatment delay.

Conclusion: During the epidemic phase of an emerging infectious disease, we found that COVID-19 has several other effects besides its incidence. The postponing treatment was the most common, therefore, treatment of ovarian tumors and ovarian cancer was considered to be the most likely to be affected among gynecological diseases. Protocols that allow for easy over-diagnosis can be disadvantageous, mainly because of treatment delays, and therefore, the protocols must be developed in light of the local infection situation.
\end{abstract}

Keywords: Ovarian cancer, Ovarian tumor, Cervical cancer, COVID-19, SARS-CoV-2, Universal screening, RT-PCR, CT

\footnotetext{
*Correspondence: kobax@a2.keio.jp

Department of Obstetrics \& Gynecology, Keio University School of Medicine,

35 Shinanomachi, Shinjuku-ku, 160-8582 Tokyo, Japan
}

(C) The Author(s). 2020 Open Access This article is licensed under a Creative Commons Attribution 4.0 International License, which permits use, sharing, adaptation, distribution and reproduction in any medium or format, as long as you give appropriate credit to the original author(s) and the source, provide a link to the Creative Commons licence, and indicate if changes were made. The images or other third party material in this article are included in the article's Creative Commons licence, unless indicated otherwise in a credit line to the material. If material is not included in the article's Creative Commons licence and your intended use is not permitted by statutory regulation or exceeds the permitted use, you will need to obtain permission directly from the copyright holder. To view a copy of this licence, visit http://creativecommons.org/licenses/by/4.0/ The Creative Commons Public Domain Dedication waiver (http://creativecommons.org/publicdomain/zero/1.0/) applies to the data made available in this article, unless otherwise stated in a credit line to the data. 


\section{Background}

Since the first case of novel coronavirus disease 2019 (COVID-19) was reported in Tokyo on January 24, 2020 [1], the number of cases has continued to rise [2]. "A state of emergency" was declared from April 7 to May 25,2020 . The total number of cases exceeded 5000 on May 15, 2020.

As of June 30, 2020, 6225 infections have been reported in Tokyo, which has a population of approximately 20 million people [2]. Our hospital is located in the center of Tokyo, and it focuses on the treatment of gynecological malignancies, including ovarian cancer. We perform approximately 1200 surgeries a year, of which approximately 550 are surgeries for malignancy. At the end of March 2020, an outbreak of nosocomial infections among junior residents occurred, but no nosocomial infections occurred among gynecological inpatients. On April 8, 2020, the hospital began screening via pre-admission reverse transcriptase-polymerase chain reaction (RT-PCR) for severe acute respiratory syndrome coronavirus 2 (SARS-CoV-2) in-house. Chest computed tomography $(\mathrm{CT})$ was also performed in scheduled surgical cases with general anesthesia. Patients requiring emergency admission were managed in private rooms, and after the possibility of COVID-19 was eliminated, including negative RT-PCR results, isolation management was halted. Patients with related symptoms such as fever, cough, and taste disorder at the time of emergency admission also underwent chest $\mathrm{CT}$.

Two asymptomatic RT-PCR-positive patients were receiving treatment for gynecological malignancies. One patient with cervical cancer was followed up in the isolation ward for inpatient management, and the other patient with ovarian cancer was followed up at home. Both patients were confirmed to be RT-PCR-negative without any disease development. Although none of the patients exhibited apparent pneumonia, a few cases, including ovarian tumors or cancers, of postponed or changed treatment were prompted by the screening tests.

During an emerging infectious disease pandemic, the likelihood of being infected, as well as the disease itself, affects clinical decision making in a variety of ways. Especially, treatment strategies for ovarian tumors and cancers seemed to be more susceptible to the impact of the COVID-19 epidemic among gynecological diseases. We have summarized and presented our experience.

\section{Case presentation}

After the introduction of RT-PCR as a pre-admission screening modality in the gynecology department, RTPCR and CT were performed in 200 and 76 patients, respectively, and 20 patients who admitted emergently required RT-PCR for release from isolation, as of June 30, 2020.
Only two patients were RT-PCR-positive as mentioned previously, but a few patients had abnormal findings on chest CT. The sensitivity of RT-PCR is reported as $70 \%$, and the elimination of COVID-19 as a possible diagnosis was difficult at that time; consequently, subsequent treatment was affected.

\section{Case 1: 49-year-old woman, asymptomatic RT-PCR- positive}

The patient had stage IIIB squamous cell carcinoma of the cervix. Recurrence involving para-aortic lymph node metastasis and peritoneal dissemination was noted 9 months after concurrent chemoradiotherapy (CCRT). She was planned to participate in a randomized clinical trial of immune check point inhibitor with cytotoxic drugs for the recurrent disease. During prehospitalization screening for her first scheduled treatment, she tested positive for COVID-19 via RT-PCR. She was asymptomatic, and CT revealed no abnormal findings in the lung fields, however, left supraclavicular lymph node enlargement was observed. She was admitted to the hospital for isolation and observation for COVID-19. Repeat RT-PCR was negative on the sixth day, and the patient was discharged the following day after RT-PCR was again negative. There was no provision in the protocol of the clinical trial for COVID19 , but pre-registration tests of the trial was expired due to her hospitalization. Thus, the patient re-consented to the trail and was required to have pre-registration tests again. We started her treatment one month behind the initial schedule.

\section{Case 2: 49-year-old woman, asymptomatic RT-PCR- positive}

The patient was scheduled for surgery for an ovarian tumor. Magnetic resonance imaging (MRI) revealed a 9$\mathrm{cm}$ ovarian tumor with a solid component, suggesting the possibility of borderline malignancy or worse. Unilateral adnexal resection via laparoscopy was planned to prevent rupture/torsion and facilitate the pathological diagnosis. Preoperative RT-PCR was positive, whereas $\mathrm{CT}$ revealed no abnormalities. The patient was asymptomatic, and she was isolated at home for observation (the political policy has changed from the aforementioned example, permitting follow-up at home or in a hotel). Subsequently, the patient was confirmed negative via RT-PCR after 1 and 2 weeks, and surgery was planned again. The final pathological diagnosis was a benign lesion, but surgery was delayed by 4 weeks.

\section{Case 3: 50-year-old woman}

The patient was scheduled for surgery for an ovarian tumor. MRI identified a 5-cm ovarian enlargement that was diagnosed as mucinous cyst adenoma. Surgery was 
planned to prevent torsion and diagnose the pathology. The patient was asymptomatic and RT-PCR-negative, but CT uncovered ground-glass opacity (GGO) in the bilateral inferior lung fields (Fig. 1a). The results, although non-specific, were consistent with early COVID19, and the patient was retested after 2 weeks. During the observation, the patient progressed without any onset of disease, and CT was repeated after 2 and 3 weeks, with no change in GGO (Fig. 1b). Judging from the patient's history, the possibility of COVID-19 was eliminated, and a diagnosis of stale inflammatory changes was made. The original treatment plan was delayed by 3 weeks.

Case 4: 51-year-old woman (this case was reported before as a preliminary report [3])

The patient had stage IIIC serous carcinoma of the ovaries complicated by deep vein thrombosis and diabetes mellitus. She presented to the clinic with a chief complaint of abdominal distension, and a thorough examination revealed the aforementioned diagnosis. On pretreatment examination, deep venous thrombus was noted, and ascitic fluid retention was evident, which led to the use of neoadjuvant chemotherapy. A tri-weekly regimen of paclitaxel $180 \mathrm{mg} / \mathrm{m}^{2}$, carboplatin AUC 5, and bevacizumab $15 \mathrm{mg} / \mathrm{kg}$ was started. CT at the end of three cycles revealed shrinkage of the primary tumor and retroperitoneal lymph node metastasis (Fig. 2a), a reduction in peritoneal disseminated lesions, and a decrease in ascites; however, the new appearance of GGO, infiltrative shadows, and a rounded morphology in the bilateral middle and lower lung fields were noted (Fig. 3a). The CT findings were highly suspicious of COVID-19, but RT-PCR returned negative results. Chemotherapy was temporarily discontinued, and 2 weeks later, CT was repeated. Infiltrative shadows with fibrosis were observed in both inferior lung fields, which was consistent with the resolution of pneumonia (Fig. $3 \mathrm{~b})$. Repeat CT was performed 2 weeks later, and the GGO tended to dissipate (Fig. 3c). However, swelling of the retroperitoneal lymph nodes had returned. Repeat CT 2 weeks later confirmed no change in the pneumonia. Ascites had worsened, and the retroperitoneal lymph nodes were larger (Fig. 2b). RT-PCR was performed, and the results were again negative. The possible causes of pneumonia were COVID-19 and druginduced pneumonia. However, even if COVID-19 was present, the disease was not active, and it was determined that chemotherapy would take priority because of exacerbation of the primary disease. Considering the possibility of drug-induced pneumonia, the drug was changed from paclitaxel to liposomal doxorubicin, and chemotherapy was resumed. Treatment was delayed by 7 weeks in this case, and the delay resulted in a clinically evident exacerbation of the case.

\section{Case 5: 58-year-old woman}

The patient had stage IIIB adenosquamous carcinoma of the cervix, and recurrence with re-growth of the primary tumor was noted 2 months after CCRT. Because of tumor-induced hydronephrosis, bilateral ureteral stents were indwelled.

She was admitted to the hospital on an emergency basis because of back pain and deterioration of kidney function. Multiple bone metastases and pathological fractures were noted on CT on admission, but simultaneously, GGO and a granular shadow were noted in the right upper lobe of the lung field, suggesting the possibility of COVID-19 (Fig. 4). RT-PCR was performed the next day, and the results were negative. There were no related symptoms such as fever or respiratory symptoms other than pain. On the fourth day of hospitalization,

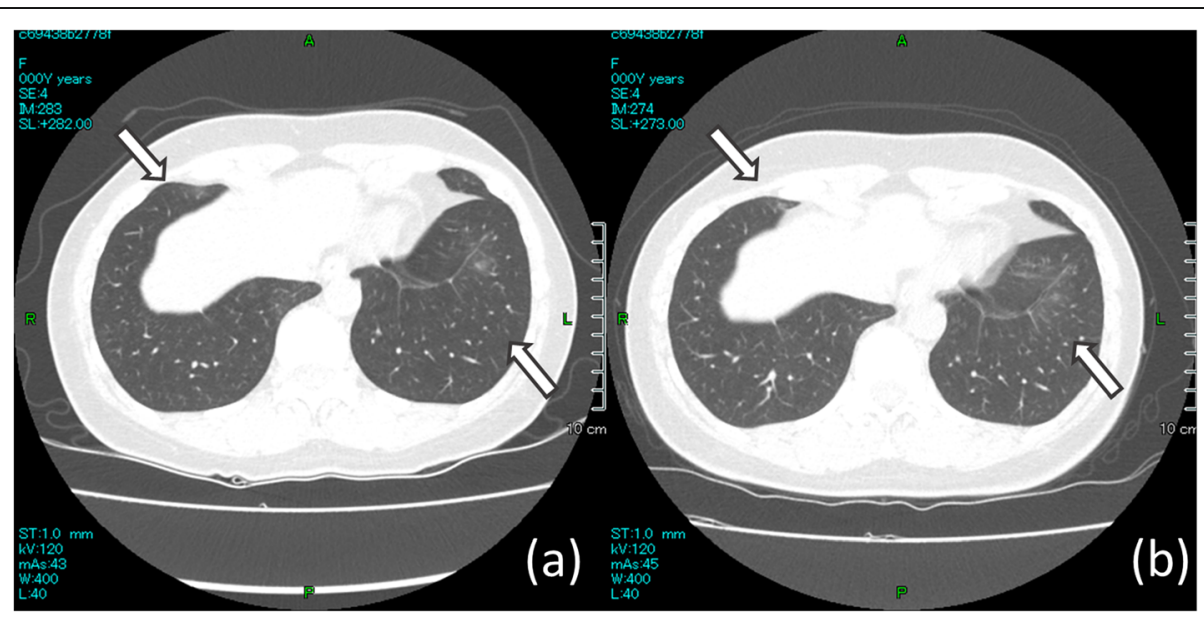

Fig. 1 Computed tomography (CT) images of the lung field of Case 3. At the time of initial presentation (a) and 3 weeks later (b). CT revealed ground-glass opacity in the bilateral inferior lung fields (arrows) but no change over time 


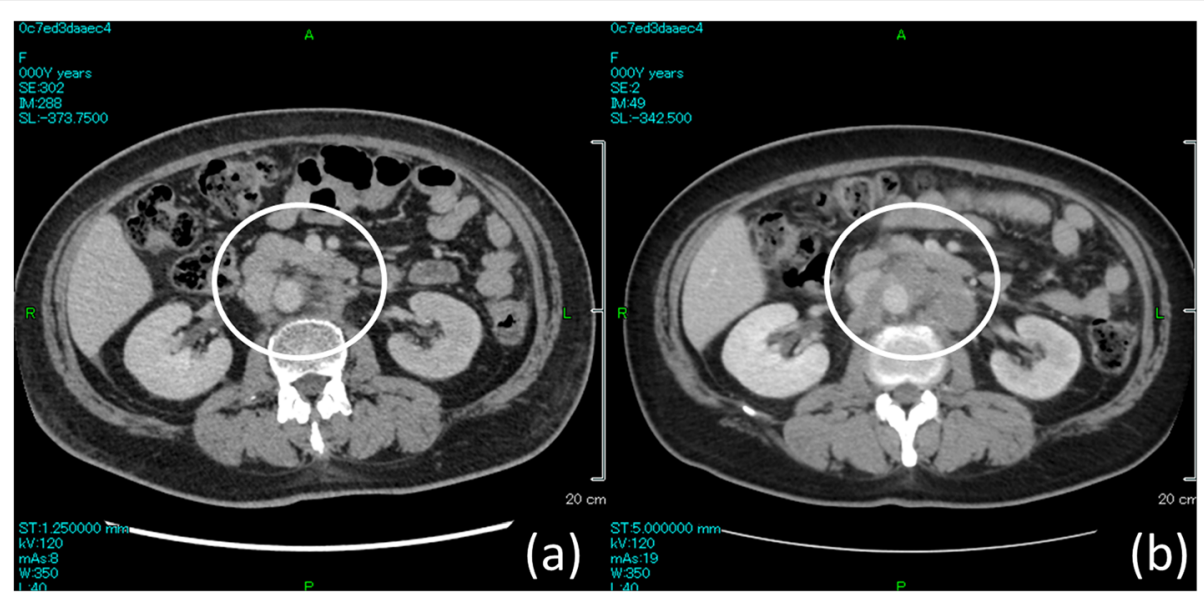

Fig. 2 Abdominal computed tomography (CT) images of Case 4. The metastatic para-aortic lymph nodes (in circles) had shrunk after three cycles of chemotherapy (a), but re-growth occurred during the delay in treatment (b)

RT-PCR was repeated with negative results, and the patient was released from isolation to a private room. Bone metastases were irradiated, and the patient's pain was controlled with opioids. In addition, urinary tract obstruction and infection were treated, the patient's renal function improved, and she was discharged after 1 month of hospitalization. Before discharge, the patient was re-examined via $\mathrm{CT}$, and although $\mathrm{GGO}$ in the upper lung field was exacerbated, the likelihood of morbidity was low. Therefore, the patient was considered to have other conditions such as atypical pneumonia or drug-induced pneumonia. The situation required inpatient management, regardless of the suspected COVID-19, to control other symptoms and conditions. Although the only medical issue was the need for isolation for infection control during hospitalization, it was possible that a suspected infection during the end-of-life care may have caused emotional distress. In fact, the patient was able to stay at home for 2 weeks, she was later re-admitted to the hospital, at which she died of sepsis. If the suspicion of COVID-19 was not resolved, the quality of life during end-of-life case could have been diminished because of the lack of time with family and adequate palliative care.

\section{Discussion and conclusion}

\section{Difficulty in deciding whether to postpone treatment}

The global spread of COVID-19, an emerging infectious disease, has exerted a variety of effects on high-volume gynecological oncology practices, and these effects were attributable to both the disease itself and the suspicion of disease. In most cases, the mere postponement of a necessary scheduled treatment may have a significant impact, but in the case of cancer treatment, the impact of the treatment delay represents a therapeutic disadvantage.

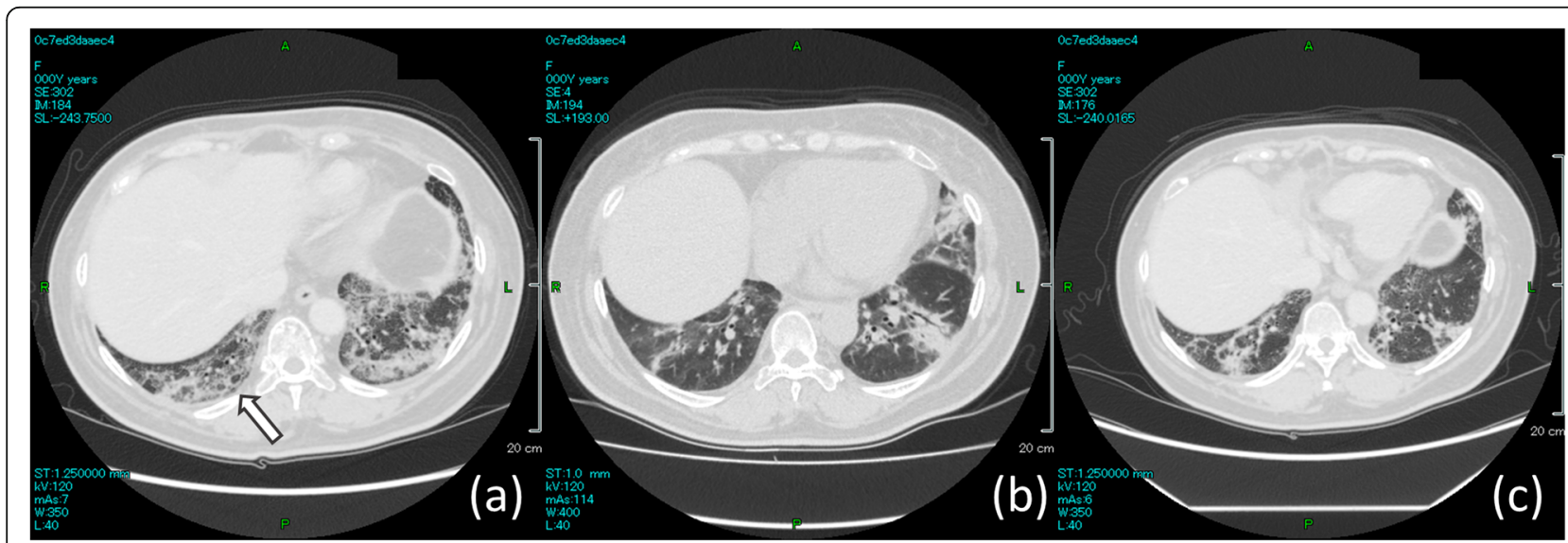

Fig. 3 Computed tomography (CT) images of the lung field of Case 4. CT revealed the new appearance of ground-glass opacity, infiltrative shadows, and a rounded morphology (arrow) in the bilateral middle and lower lung fields (a). Fibrosis developed within 2 weeks (b) and then tended to disappear (c). The findings were highly suspicious of coronavirus disease 2019. Figure 3a was reprinted from reference [3] 


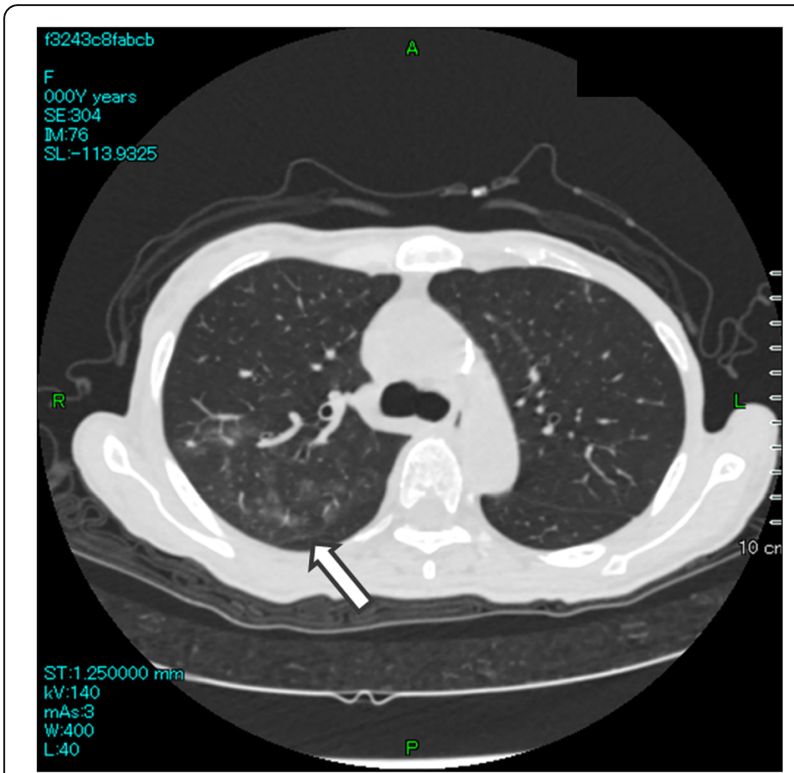

Fig. 4 Computed tomography $(\mathrm{CT})$ images of the lung field of Case 5. CT revealed ground-glass opacity and granular shadows in the right upper lobe of the lung field (arrow)

In the case of surgery, it is desirable to conduct the operation as soon as possible before the disease progresses. In the case of chemotherapy, if treatment is discontinued temporarily, then the dose intensity will be reduced. For instance, for six cycles of tri-weekly paclitaxel and carboplatin therapy, if COVID-19 causes a 4week delay during treatment, the relative dose intensity will be approximately $82 \%$. The relationship between the relative dose intensity and prognosis has been described for various cancer types [4-9]. Even if treatment is deferred, it is not desirable for treatment to be postponed unnecessarily. In addition to the physical therapeutic disadvantages, delays in planned treatment were reported to cause anxiety and depression for patients, leading to lower quality of life [10].

Conversely, patients with cancer are especially likely to have a high risk of severe COVID-19 [11, 12]. In addition, surgery in patients with COVID-19, even in those without coincident cancer, may result in miserable outcomes [13-15]. Because of the limited sensitivity of RT-PCR, treatment decisions must be made with caution.

The recommendation for suspension of chemotherapy in patients with COVID-19 is considered reasonable, and a delay of 2-4 weeks is currently recommended [16]. A patient with ovarian cancer was reported to have safely resumed chemotherapy in COVID-19 convalescence with a similar withdrawal period [17]. However, it is unclear whether the required duration of treatment interruption is similar between patients with apparent pneumonia and asymptomatic RT-PCR-positive patients.
There was a report of a patient who tested positive antibodies during chemotherapy. Although it was unclear at what point she contracted, the patient was able to continue chemotherapy safely [18].

\section{Decision based on CT findings}

Regarding imaging, typical CT findings of COVID-19 are said to be peripheral, inferior, and bilateral, starting with bilateral peripheral frosted shadows in the early stages and extending to tuck the lobes of the lungs during disease progression. However, it has been reported that more than half of patients have normal findings in the early stages of the disease $[19,20]$. Thus, normal findings on CT do not eliminate the possibility of COVID-19 in patients with a suspicious history and symptoms.

Meanwhile, a meta-analysis uncovered a specificity of $37 \%$ for CT [21]. If there are only abnormalities in the CT findings, different diagnoses are more likely. Because little remains known about COVID-19, we have concerns regarding the worst consequences of forcing treatment. Because of this cautiousness, treatment is often delayed, and judgment is withheld until follow-up and repeat RT-PCR confirm the absence of COVID-19.

\section{Susceptibility of the treatment strategies for ovarian tumors and ovarian cancer}

Given the above two discussions, it would be difficult to diagnose accurately during an epidemic. When we allowed some over-diagnosis, there would be more cases where treatment is discontinued. This would most likely affect the treatment of ovarian tumors and ovarian cancer (including fallopian tube and peritoneal cancers). Three of the five cases in the present study were ovarian tumors and ovarian cancer.

The first reason is that in many ovarian tumors, the surgeries are required for pathological diagnosis. Once a diagnosis of malignancy is made in an outpatient examination, such as cervical or uterine cancer, the priority of treatment would be increased. In the worst-case scenario, such as surgical restrictions or hospital closures, the patient could be transferred to another gynecological malignancy treatment facility. Patients who are not suspected of having aggressive malignant findings on imaging may be treated as benign and put off. Case 2 and 3 in this series had resulted in benign histology, but if a malignant, significant delay in treatment.

The second reason is that in advanced cases of ovarian cancer, the treatment plan of neoadjuvant chemotherapy (NAC) followed by interval debulking surgery (IDS) is more often chosen than cervical or uterine cancer. IDS requires careful planning in advance, such as anticipating the duration of bone marrow recovery from the latest chemotherapy, but deferral due to COVID-19 could 
suddenly ruin that plan. In case 4 , the patient was suspected of having COVID-19, and while the chemotherapy was postponed, the patient showed worsening of the disease and did not reach IDS.

This issue had been discussed, not just from our own experiences. Guidelines and recommendations have been issued by various organizations, including countries with more widespread conditions, such as China, Italy, and the United States [22, 23]. According to these guidelines, CCRT for cervical cancer was recommended with high priority, and many patients could receive standard treatment. For advanced ovarian cancer, there were recommendations to avoid highly invasive primary debulking surgery, which is likely to occupy the intensive care unit. Thus, a strategy of neoadjuvant chemotherapy to interval debulking surgery would be recommended, but this, of course, needs to be balanced with individual patient prognosis, which is a difficult decision [24, 25]. Surgery for endometrial cancer was also considered a low priority for early-stage cancer [23]. Ovarian cancer is the most susceptible among the three major gynecological cancers, considering the prognoses and the rate of advanced cancer [26].

\section{Preventive measures for patients with cancer during hospital closures caused by nosocomial infections}

In response to the spread of epidemics in Japan, the Japanese Society of Obstetrics and Gynecology has created a network system, named Perinatal Early Assessment and Communication system for Emergencies, for sharing information about hospital functioning in real-time for hospitals across the country [27]. This system was established for disaster situations, but it is intended for perinatal care only. There is no similar system for the treatment of gynecological malignancies, and if most hospitals are shut down because of the nosocomial spread of COVID-19, many patients may be unable to obtain treatment. In fact, because of the COVID-19 epidemic in Tokyo, other high-volume gynecological malignancy treatment facilities in the city have temporarily limited medical functions [28]. We have established an information-sharing network at 19 hospitals in Tokyo (Gynecologic oncological surgery communication in Tokyo in New Infection pandemic by Coronavirus; URL undisclosed). As of June 30, 2020, although the epidemic has not yet resulted in the transfer of patients to other hospitals en masse, we are preparing for a large surge in the number of patients seeking treatment.

\section{Sensitivity, specificity, benefits, and drawbacks of universal testing based on the epidemic situation}

Because nosocomial infections were identified among junior clinical residents of our hospital staff [29], the executive committee, as a prudent response, implemented a screening system using RT-PCR and CT for all patients before admission, resulting in possible overdiagnosis. As a result, we found that approximately $6 \%$ of asymptomatic individuals were RT-PCR-positive in the week of April 13-19, 2020 [30]. Based on previous reports, the sensitivity and specificity of RT-PCR were 70 and $100 \%$, respectively [31], and those of CT are 94 and $37 \%$ [21]. Thus, in a population that is $6 \%$ RT-PCRpositive, the positive predictive value and negative predictive value of RT-PCR are 100 and $97.3 \%$, respectively, and those of CT are 12.3 and $98.5 \%$, respectively. Indeed, a screening program is likely to be a solid barrier to preventing the admission of unrecognized infected people. However, because a positive CT finding alone requires treatment delay and follow-up, approximately $90 \%$ of patients with a positive CT finding may experience an unnecessary delay in treatment. Testing should be selected on the basis of the local infection situation. Because the emergency declaration was lifted and the infection rate in the general population was believed to have decreased further, preoperative screening using CT was discontinued on June 22, 2020.

To date, the factors that predict severe disease for asymptomatic and mildly ill individuals have been identified [32], but it is hoped that investigation concerning the presence or absence of true infection for asymptomatic individuals will continue [31].

During the epidemic phase of an emerging infectious disease, we found that the disease has several other effects besides its incidence. The postponing treatment was the most common, therefore, treatment of ovarian tumors and ovarian cancer was considered to be the most likely to be affected among gynecological diseases. Protocols that allow for easy over-diagnosis can be disadvantageous, mainly because of to treatment delays, and therefore, protocols must be developed in light of the local infection situation.

\section{Abbreviations \\ CCRT: Concurrent chemoradiotherapy; GGO: Ground-glass opacity}

\section{Acknowledgements \\ We would like to thank the infection control team and medical technologists for their efforts in establishing our screening test system.}

\section{Authors' contributions}

YN obtained the consent for publication, collected data, and contributed to the drafting of the manuscript. All authors are members of gynecological oncology team deciding management and attending patient care. Among them, MN manages the surgery schedule and TM manages the hospital beds. YN, YK, KB, and DA are the core members who developed the concept of this report. All authors read and approved the final manuscript.

Funding

This study received no sources of funding.

Availability of data and materials

All data used during this study are included in this published article. 


\section{Ethics approval and consent to participate}

The Keio University School of Medicine Ethical Committee approved the research and the publication of clinical information for patients with gynecological benign disease (approval number: 20150105) and malignancy (approval number: 20070081).

\section{Consent for publication}

Consent for publication has been obtained for all patients in this report.

\section{Competing interests}

The authors declare that they have no competing interests.

Received: 10 August 2020 Accepted: 1 September 2020

Published online: 11 September 2020

\section{References}

1. Tokyo Metropolitan Government. https://www.metro.tokyo.lg.jp/tosei/ hodohappyo/press/2020/01/24/20.html. Accessed 30 Jun 2020.

2. Tokyo Metropolitan Government COVID-19 information website. https:// stopcovid19.metro.tokyo.lg.jp/cards/number-of-confirmed-cases. Accessed 30 Jun 2020

3. Kobayashi Y, Suh DH, Aoki D, Kim JW. Management of ovarian cancer patients in affected areas during COVID-19 pandemic: Japan and Korea. J Gynecol Oncol. 2020;31(3):e65.

4. Levin L, Simon R, Hryniuk W. Importance of multiagent chemotherapy regimens in ovarian carcinoma: dose intensity analysis. J Natl Cancer Inst. 1993:85(21):1732-42

5. Fauci JM, Whitworth JM, Schneider KE, Subramaniam A, Zhang B, Frederick PJ, et al. Prognostic significance of the relative dose intensity of chemotherapy in primary treatment of epithelial ovarian cancer. Gynecol Oncol. 2011:122(3):532-5.

6. Hanna RK, Poniewierski MS, Laskey RA, Lopez MA, Shafer A, Van Le L, et al. Predictors of reduced relative dose intensity and its relationship to mortality in women receiving multi-agent chemotherapy for epithelial ovarian cancer. Gynecol Oncol. 2013;129(1):74-80.

7. Denduluri N, Patt DA, Wang Y, Bhor M, Li X, Favret AM, et al. Dose delays, dose reductions, and relative dose intensity in patients with Cancer who received adjuvant or Neoadjuvant chemotherapy in community oncology practices. J Natl Compr Canc Netw. 2015:13(11):1383-93.

8. Denduluri N, Lyman GH, Wang Y, Morrow PK, Barron R, Patt D, et al. Chemotherapy dose intensity and overall survival among patients with advanced breast or ovarian Cancer. Clin Breast Cancer. 2018:18(5):380-6.

9. Crawford J, Denduluri N, Patt D, Jiao X, Morrow PK, Garcia J, et al. Relative dose intensity of first-line chemotherapy and overall survival in patients with advanced non-small-cell lung cancer. Support Care Cancer. 2020;28(2): 925-32.

10. Frey MK, Ellis AE, Zeligs K, Chapman-Davis E, Thomas C, Christos PJ, et al. Impact of the coronavirus disease 2019 pandemic on the quality of life for women with ovarian cancer. Am J Obstetrics Gynecol. 2020;S0002-9378(20): 30674-8.

11. Tang LV, Hu Y. Poor clinical outcomes for patients with cancer during the COVID-19 pandemic. Lancet Oncol. 2020;21(7):862-4

12. Zhang L, Zhu F, Xie L, Wang C, Wang J, Chen R, et al. Clinical characteristics of COVID-19-infected cancer patients: a retrospective case study in three hospitals within Wuhan, China. Ann Oncol. 2020;31(7):894-901.

13. Aminian A, Kermansaravi M, Azizi S, Alibeigi P, Safamanesh S, Mousavimaleki A, et al. Bariatric surgical practice during the initial phase of COVID-19 outbreak. Obes Surg. 2020;30(9):3624-7.

14. Aminian A, Safari S, Razeghian-Jahromi A, Ghorbani M, Delaney CP. COVID-19 outbreak and surgical practice: unexpected fatality in perioperative period. Ann Surg. 2020;272(1):e27-9.

15. Nahshon C, Bitterman A, Haddad R, Hazzan D, Lavie O. Hazardous postoperative outcomes of unexpected COVID-19 infected patients: a call for global consideration of sampling all asymptomatic patients before surgical treatment. World J Surg. 2020;44(8):2477-81.

16. American Society of Clinical Oncology. General Information about COVID-19 \& Cancer. https://www.asco.org/asco-coronavirus-resources/care-individualscancer-during-covid-19/general-information-about-covid-19. Accessed 30 Jun 2020
17. Liontos M, Kaparelou M, Karofylakis E, Kavatha D, Mentis A, Zagouri F, et al. Chemotherapy resumption in ovarian cancer patient diagnosed with COVID-19. Gynecol Oncol Rep. 2020;33:100615.

18. Chen Y, Li G. Gynecological malignancies with asymptomatic SARS-CoV2 infection during the convalescence of outbreak. Gynecol Oncol. 2020; 158(1):44-6.

19. Bernheim A, Mei X, Huang M, Yang Y, Fayad ZA, Zhang N, et al. Chest CT findings in coronavirus Disease-19 (COVID-19): relationship to duration of infection. Radiology. 2020;295(3):200463.

20. Zhao W, Zhong Z, Xie X, Yu Q, Liu J. Relation between chest CT findings and clinical conditions of coronavirus disease (COVID-19) pneumonia: a multicenter study. AJR Am J Roentgenol. 2020;214(5):1072-7.

21. Kim H, Hong H, Yoon SH. Diagnostic Performance of CT and Reverse Transcriptase-Polymerase Chain Reaction for Coronavirus Disease 2019: A meta-analysis. Radiology. 2020. https://doi.org/10.1148/radiol.2020201343.

22. Wang $Y$, Zhang $S$, Wei $L$, et al. Recommendations on management of gynecological malignancies during the COVID-19 pandemic: perspectives fromChinese gynecological oncologists. J Gynecol Oncol. 2020;31(4):e68. https://doi.org/10.3802/jgo.2020.31.e68.

23. Uwins C, Bhandoria GP, Shylasree TS, et al. COVID-19 and gynecological cancer: a review of the published guidelines [published online ahead of print,2020 Jun 23]. Int J Gynecol Cancer. 2020;ijgc-2020-001634. https://doi. org/10.1136/ijgc-2020-001634.

24. Bogani G, Casarin J. Management of patients with ovarian cancer in the COVID-19 era. 2020;122(2):122-3.

25. Mandato VD, Aguzzoli L. Management of ovarian cancer during the COVID19 pandemic. Int J Gynaecol Obstetrics. 2020;149(3):382-3.

26. Nagase $\mathrm{S}$, Ohta T, Takahashi F, Enomoto T. Annual report of the committee on gynecologic oncology, the Japan Society of Obstetrics and Gynecology: annual patients report for 2015 and annual treatment report for 2010. J Obstet Gynaecol Res. 2019;45(2):289-98.

27. Japan Society of Obstetrics and Gynecology. Perinatal Early Assessment and Communication system for Emergencies: PEACE. http://www.jsog.or.jp/ modules/disaster/index.php?content_id=1. Accessed 30 Jun 2020.

28. Asahi Shimbun Digital on $14^{\text {th }}$ Apr.https://www.asahi.com/articles/ASN4 F7DSNN4FUTIL02K.html?iref=pc_ss_date. Accessed 30 Jun 2020.

29. Keio University Hospital. http://www.hosp.keio.ac.jp/en/oshirase/detail/401 74. Accessed 30 Jun 2020.

30. Asahi Shimbun Digital on 23th Apr. http://www.asahi.com/ajw/ articles/13321354. Accessed 30 Jun 2020.

31. Woloshin S, Patel N, Kesselheim AS. False negative tests for SARS-CoV-2 infection - challenges and implications. N Engl J Med. 2020;383(6):e38.

32. Gandhi RT, Lynch JB, Del Rio C. Mild or moderate Covid-19. N Engl J Med. 2020

\section{Publisher's Note}

Springer Nature remains neutral with regard to jurisdictional claims in published maps and institutional affiliations.

Ready to submit your research? Choose BMC and benefit from

- fast, convenient online submission

- thorough peer review by experienced researchers in your field

- rapid publication on acceptance

- support for research data, including large and complex data types

- gold Open Access which fosters wider collaboration and increased citations

- maximum visibility for your research: over $100 \mathrm{M}$ website views per year

At $\mathrm{BMC}$, research is always in progress.

Learn more biomedcentral.com/submission 